\title{
Wireless sensor system for monitoring spatially resolved tongue pressure
}

\author{
Stöhr, Ingmar \\ TU Darmstadt, Institut EMK \\ Merckstr. 25, 64283 Darmstadt
}

\begin{abstract}
In this paper we describe a wireless sensor system for measuring the spatially resolved pressure of the tongue against the upper palate. The system consists of eight piezoresistive absolute pressure sensors, a signal conditioning and data acquisition electronics, a wireless transceiver module and a LiPo-battery. A novel encapsulation protects the sensing elements during the manual mounting process, while taking into account the sensibility of the tongue. The implemented technique of switched excitation voltage to reduce the power consumption of the sensors by $97 \%$ is discussed. The design of the signal conditioning and data acquisition electronics is presented. Tongue pressure is sampled with $30 \mathrm{~Hz}$ per sensor and with a resolution of $0.25 \mathrm{mbar}$. The sensor systems overall power consumption with working transceiver is $56 \mathrm{~mW}$, enabling a running time of more than two hours out of the integrated $60 \mathrm{mAh}$ LiPo-battery. First measurement results of the working sensor system in the oral cavity are given.
\end{abstract}

\section{Introduction}

Orthodontists are interested in the position and pressure of the tongue against the hard palate since it has influence on many functions like speaking, swallowing and the growth of the mandible. In the past some efforts were made to measure the tongue pressure and its spatial distribution. But all designs incorporate wires or tubes for connecting the intra oral measuring device to the extra oral data acquisition electronics, thus disturbing the natural function of tongue and lips. Main goal of the developed sensor system is to minimize the disturbance of the patient by the measurement incident. Therefore, a wireless data transmission and a data acquisition circuit have to be integrated into the intra oral device. Sensors and electronics in the area of the hard palate have to be as thin as possible.

\section{Requirements}

Measurement of tongue pressure is not a common measurement task. Tongue pressure is a distributed force, normal to the palate and can therefore be seen as pressure comparable e.g. to the force distribution between foot and floor. Contrary to typical pressure sensor application there are direct mechanical loads applied on the sensors. It can not be guaranteed, that the applied load is always isostatic. That poses restrictions on the packaging of the sensors. Further requirements evolve from the request for minimal interference with the normal behaviour of the patients tongue. Table 1 lists some of the important requirements regarding the wireless sensor system.

Preliminary tests have shown that during swallowing and speech relevant pressure amplitudes are present up to $15 \mathrm{~Hz}$. Although for analysis of speech disorders it can be of interest to expand the frequency range. Therefore, the minimal sampling rate of the system is to be $30 \mathrm{~Hz}$ per sensor with the option of higher sample rates. Due to the fact, that the system has to operate wirelessly, a power supply, signal conditioning and data acquisition electronics and a transceiver have to be integrated in the intra oral device.

Table 1: Requirements for the wireless measurement system

\begin{tabular}{|l|l|l|}
\hline Requirement & Value & Comment \\
\hline measuring range & $-50 \ldots+200 \mathrm{mbar}$ & relative to ambient pressure \\
\hline min. resolution & $1 \mathrm{mbar}$ & \\
\hline max. thickness & $1,5 \mathrm{~mm}$ & better 1 mm \\
\hline frequency range & static ... 15 Hz & higher sample rates favourable \\
\hline min. number of sensors & 8 & \\
\hline
\end{tabular}




\section{Measurement system}

Below the developed wireless sensor system is presented with focus on the used sensing elements, their packaging and the signal conditioning and data acquisition electronics. The layout was chosen according to previous research at our institute [1]. Figure 1 shows a view of the upper palate with the key components of the measurement system and their positions. The eight pressure sensors are placed in the area of tongue-contact. They are connected via flexible PCBs to the sensor electronics, which is placed right behind the tongue-contact region to assure short connections. Power supply and telemetry module are placed in the cheek pouches, where their influence on tongue function is negligible.

All components are mounted in a palatal plate made individually for each patient of thermoplastic by vacuum forming. The flexible PCB connections offer advantages over the insulated copper wires used in [1]. The whole system, including electronics and sensing elements can be mounted on one flexible PCB prior to mounting it into the palatal plate, thus reducing the number of solder joints to a minimum. The mounting of the measurement system can then be done by a dental technician since no further work on electronics is required.

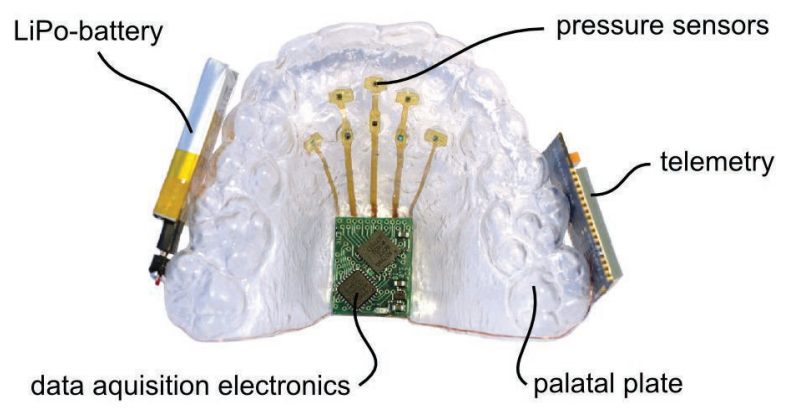

Fig. 1: Wireless sensor system for measuring the spatially resolved tongue pressure.

\subsection{Sensing elements}

As sensing elements piezoresistive silicon absolute pressure sensors are used. Due to mechanical loads on the palatal plate the pressure on is backside varies. Therefore, the use of absolute pressure elements is necessary. Opposite to differential pressure sensors, their closed design makes sterilization of the measurement system easier.

Due to their lower power consumption capacitive sensing elements would be preferable compared to resistive sensors. However they need the signal conditioning close up or preferably on the sensing element itself. Capacitive sensing elements with integrated signal conditioning are common in research but are not freely available in the required dimensions.

On the contrary piezoresistive sensing elements are available in miscellaneous measuring ranges and designs. And the disadvantage of higher power consumption can be overcome with the right kind of sensor electronics, as will be shown below.

Thus the smallest commercially available piezoresistive absolute pressure sensing element is chosen for the measurement system. Type SM5108C from Silicon Microstructures, USA, is a classically designed sensing element with dimensions of $0.65 \times 0.65 \times 0.65 \mathrm{~mm}^{3}$.

\subsection{Packaging of the sensing elements}

The sensing elements are mounted on flexible PCBs. Contacting is done by means of wedge-wedge bonding with $25 \mu \mathrm{m}$ aluminium wires. Contrary to common pressure sensor applications tongue pressure is not an isostatic load with a gas or fluid, but a direct and sometimes partial mechanical load with a more or less rigid object. This and the fact, that the sensor system has to be mounted manually and individually has to be taken account for. On one hand the packaging has to protect the bond wires during the mounting process and also during normal use. On the other hand its influence on sensor characteristics has to be kept as low as possible.

In [2] a two stage polymeric package is suggested. A hard (Shore D80) UV-curing epoxy resin (Vitralit 1605 ) is applied around the sensing element and under the bond wires. After curing the bond wires are much more robust to touch and the flex PCB together with the sensing element is mounted trough holes in palatal plate. In a following step the holes are filled with a soft (Shore A55) UV-curing acrylic urethane. By doing that, everything is encapsulated watertight and a smooth transition from encapsulation to palatal plate is achieved. This is important, because the tongue is very sensitive to hard edges and small gaps. 
The encapsulation reduces the sensitivity of the sensors by only 5 to $6.5 \%$. It can protect the sensing elements from water for one hour with only $0.5 \%$ change in sensitivity. But afterwards the encapsulated sensors need several days of drying to return to normal characteristics. As to be expected the encapsulation introduces creep of up to $0.6 \%$ of full scale after 240 seconds and low pass behaviour, which still has to be characterised in detail. A detailed description of the package and its characteristics can be found in [3].

\subsection{Power consumption of the sensors}

As mentioned earlier, power consumption is one weakness of resistive sensors. The incorporated sensing elements possess a typical bridge resistance of $5 \mathrm{k} \Omega$. At an excitation voltage of $3.3 \mathrm{~V}$ each sensor will consume $2.18 \mathrm{~mW}$ meaning $17.4 \mathrm{~mW}$ for eight sensors. Table 2 shows the power consumptions of the main components.

Table 2: Power consumption of main components of the wireless measurement system.

\begin{tabular}{|r|r|}
\hline \multicolumn{1}{|l|}{ Component } & Power Consumption \\
\hline \multicolumn{1}{|l|}{ Microcontroller (ATmega168PA) } & $7.60 \mathrm{~mW}$ \\
\hline sleep & $<0.01 \mathrm{~mW}$ \\
\hline active & $11.70 \mathrm{~mW}$ \\
\hline active & $0.01 \mathrm{~mW}$ \\
\hline sleep & $59.40 \mathrm{~mW}$ \\
\hline Telemetry (ZigBit ATZB-24-A2) & $62.70 \mathrm{~mW}$ \\
\hline transmitting at 0 dBm & $0.02 \mathrm{~mW}$ \\
\hline receiving & $17.40 \mathrm{~mW}$ \\
\hline sleep & \\
\hline Eight Pressure Sensors (SM5108C) & \\
\hline
\end{tabular}

It turns out, that the active sensors are responsible for the biggest part of the power loss besides the wireless transceiver. Therefore, the sensor electronics is designed in such a way, to allow switching excitation voltage for every sensor individually.

To get a stable and accurate measurement several effects of the switching have to be taken into account. The voltage at the input of the ADC has to be stable over the whole sampling time. That means the sensor has to be switched on for at least this time. Other limiting factors are the electrical and thermal settling times. Once connected to the power supply, various capacitances on the sensing element have to be loaded, until the output stabilizes. Then the input amplifier has to settle. The current flowing through the bridge will cause local heating leading to a thermal drift of the output until a steady state is reached.

Self heating was measured by connecting an unpackaged SM5108 sensing element to a National Instruments PXle-4330 bridge input module. The sensor output was recorded for several minutes with $1 \mathrm{ksps}$. Results show a thermal time constant of $0.46 \mathrm{~s}$. The settling function was calculated to be:

$$
v_{\text {out }}(t)=31.35 \frac{\mu \mathrm{V}}{\mathrm{V}} \cdot e^{-\frac{t}{0.46 \mathrm{~s}}}+19042 \frac{\mu \mathrm{V}}{\mathrm{V}}
$$

The error made during the sampling time due to self heating can now be calculated according to eqn. 2 .

$$
\text { error }_{\text {therm }}(t)=\frac{1-e^{-\frac{t}{0.46 \mathrm{~s}}}}{608.4}
$$

To keep the error due to self heating below one LSB for a 16 bit ADC the change has to be kept below $15 \mathrm{ppm}$. That is guaranteed for sampling times $\mathrm{t}_{\mathrm{s}}$ shorter than $4 \mathrm{~ms}$. Of course the sensors have to be calibrated in this switched mode, since the sensors will be running in a different thermal steady state as if powered continuously.

The electrical time constant $\tau_{\mathrm{e}}$ of the sensor is measured to be in the range of 45 to $75 \mathrm{~ns}$. To keep this error also below one LSB of a 16 bit ADC, the required settling time can be calculated to eleven times $\tau_{\mathrm{e}}$ according to equation 3 .

$$
-\frac{t}{\tau_{e}} \geqslant \ln \left(\frac{1}{2^{16}}\right)
$$


That means the sensor has to be powered up at least 825 ns before the sampling starts. Sampling can take up to $4 \mathrm{~ms}$ until self heating introduces an error of one LSB. In this calculation the settling time of the amplifier is not considered and settling time will also depend on the type of switching circuit.

With the designed electronics the above theory could be verified. One sensor output was measured with continuous excitation and with switched excitation voltage. The switched variant was tested with settling times of $0.01,0.5$ and $1 \mathrm{~ms}$. The sampling time was held constant at $833 \mu \mathrm{s}$. Table 3 shows the results. The continuous excitation gives a slightly lower output due to self heating and the negative temperature coefficient of sensitivity. The switched variants all show the same results within the uncertainty of measurement of $15.4 \mu \mathrm{V}$.

Table 3: Sensor output for continuous and switched excitation with different settling times.

\begin{tabular}{|r|c|}
\hline settling time (ms) & sensor output (mV) \\
\hline continuously powered & 30.306 \\
\hline 0.01 & 30.404 \\
\hline 0.50 & 30.408 \\
\hline 1.00 & 30.407 \\
\hline
\end{tabular}

\subsection{Signal conditioning and data acquisition electronics}

Since one requirement was wireless data transmission of sensor data, some kind of signal conditioning has to be placed in the oral cavity. Decision was made to use a digital transmission for ease of implementation and a better noise immunity. Therefore, the electronic has to consist of analog signal conditioning, analog to digital converter and control logic.

Space in the palatal plate is very limited. For the data acquisition electronics a space of maximum $2 \mathrm{~cm}^{2}$ can be used. The Thickness of the whole system should be below $1 \mathrm{~mm}$ for minimal disturbance of the patient. Many possible topologies for an eight channel data acquisition circuit were considered. Because integration density on a PCB is lower than on a silicon die, only highly integrated circuits can fulfil the requirements. Since development of an ASIC was not scheduled in the scope of the project, the choices are restricted to parts freely available on the market.

The best solution in this case is the AD7194 from Analog Devices. It combines a 24-bit Delta-Sigma ADC with a differential programmable gain amplifier an input multiplexer for eight differential inputs in a package of only $5 \times 5 \times 0.85 \mathrm{~mm}^{3}$. It can provide a sampling rate of $1.2 \mathrm{kHz}$ in single conversion mode.

To fulfil the Nyquist-Shannon sampling theorem the bandwidth has to be limited. In this case, even a single discrete RC low pass filter for each input would have needed at least an additional $18 \mathrm{~mm}^{2}$ on the PCB using 0201 scale SMD components. The pressure signal itself is innately bandwidth limited due to the mechanical low pass behaviour of the sensor and the encapsulation. Also the output resistance of the bridge together with stray capacitances result in an unspecified electrical low pass filter. Together with the high oversampling of the delta-sigma ADC (internal sampling rate of $5 \mathrm{MHz}$ ) it seems to be viable to discard the dedicated low pass filters. To prove this assumption right, a test circuit with and without the low pass filters was build. There was no measureable difference between both variants in regard to output value and standard derivation thereof. Therefore, the final layout was done without dedicated low pass filters.

The AD7194 is connected to an Atmel ATmega168PA microcontroller via SPI. The microcontroller also switches the excitation voltage of the sensors. The low side of each bridge is connected directly to one of the microcontrollers IO-pins. The pins are either pulled to ground or left in high-impedance mode, thus turning off the sensor. The microcontroller configures the AD7194 and controls the timing of the measurements. The ADC is configured in its fastest mode $(1200 \mathrm{kHz})$, resulting in a sampling time of $833 \mu \mathrm{s}$. One sampling cycle follows the following pattern: The first sensor is connected to the excitation voltage. Then the ADC is configured for the upcoming conversion. After the precalculated electrical settling time has passed, the conversion is started. After the conversion the sensor is turned off, the sampled data is read by the microcontroller and the next sensor is connected to the excitation voltage. This is done eight times until all sensors are sampled once. Then the acquired data is transferred to the telemetry module. This cycle is repeated with a frequency of $30 \mathrm{~Hz}$ (33.3 ms). Average power consumption in this mode of operation, including the sensors, is $6.6 \mathrm{~mW}$.

If desired, the sampling pattern can be changed by software. A sampling rate up to $150 \mathrm{~Hz}$ per sensor is theoretically possible. If even higher sampling rates are required, the number of sampled sensors can be reduced. For single sensor operation a sampling rate of $4.8 \mathrm{kHz}$ is possible. Figure 2 shows a block diagram and a picture of the described electronics. 

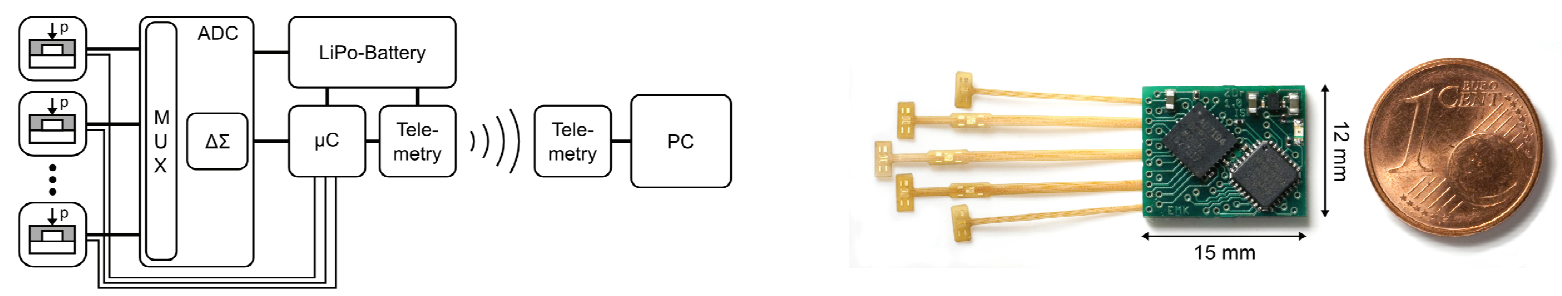

Fig. 2: Block diagram and picture of signal conditioning and data acquisition electronics. Flex PCBs for connection to sensors are mounted, but pressure sensors are missing.

\subsection{Wireless data transmission}

The wireless link has to be bidirectional in order to control the wireless system from the lab-PC, but the main communication direction is from the measurement system to a lab-PC. Eight sensors, each sampled with $30 \mathrm{~Hz}$ and 16 bit gives a data rate of $3840 \mathrm{bps}$. This is well within range of most state of the art wireless transceivers. Prior experiments and other publications show that ISM bands of $433 \mathrm{MHz}$ and $2.4 \mathrm{GHz}$ work well for data transmission out of the human body. Since space was the main limitation, small transceiver modules were investigated. The smallest, suitable module was the PAN2355 from Panasonic. It is build around a CC1100 transceiver from Texas Instruments, has data rates up to $500 \mathrm{kbps}$ and a very small size with only $8 \times 8.2 \times 2 \mathrm{~mm}^{3}$.

Due to a weak stability of the SPI-link on the PAN2355 modules a ZigBee compatible module from Atmel (ATZB-24-A2) was chosen as short term alternative. The module is significantly bigger in size $(24 \times 13.5$ $x 2 \mathrm{~mm}^{3}$ ) and the proprietary SerialNet stack running on the module limits the data rate to $38.4 \mathrm{kbps}$ on its UART interface, which increases power consumption because it takes longer for the microcontroller to transmit the data to the transceiver instead of being in sleep mode.

\section{First measurements with wireless sensor system}

A prototype of the wireless measurement system was build and first meassurements in the oral cavity were taken. Of the eight mounted sensors only four are working as expected. Based on the made expiriences this will be solved for the next prototypes. Figure 3 shows the tongue pressure during dry swallowing. Dry swallowing meaning without water or food in the mouth. Two observations can easily be made. The pressure starts building up at the foremost sensors (red, blue) and then moves backwards and to the side (green, light blue). It also turns out, that the pressure in the middle is higher than on the sides.
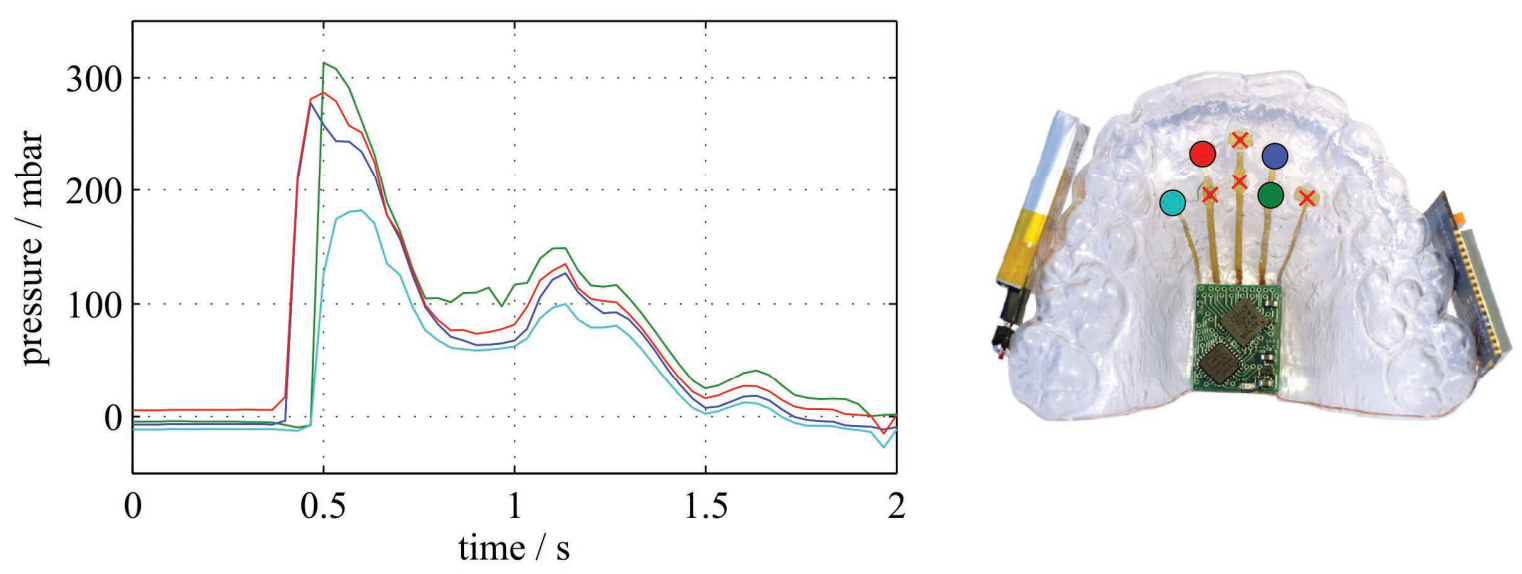

Fig. 3: Tongue pressure during dry swallowing, measured with the prototype shown on the right. Colors represent the different sensor locations as shown on the right. Non functional sensors marked with red $X$.

Figure 4 shows the tongue pressure during swallowing water. It can be clearly differentiated from Figure 3. Tongue pressure is first present at the outermost sensors, which could be interpreted as sealing the space between tongue and upper palate. Then the pressure builds up on the inner sensor while the water is swallowed. 

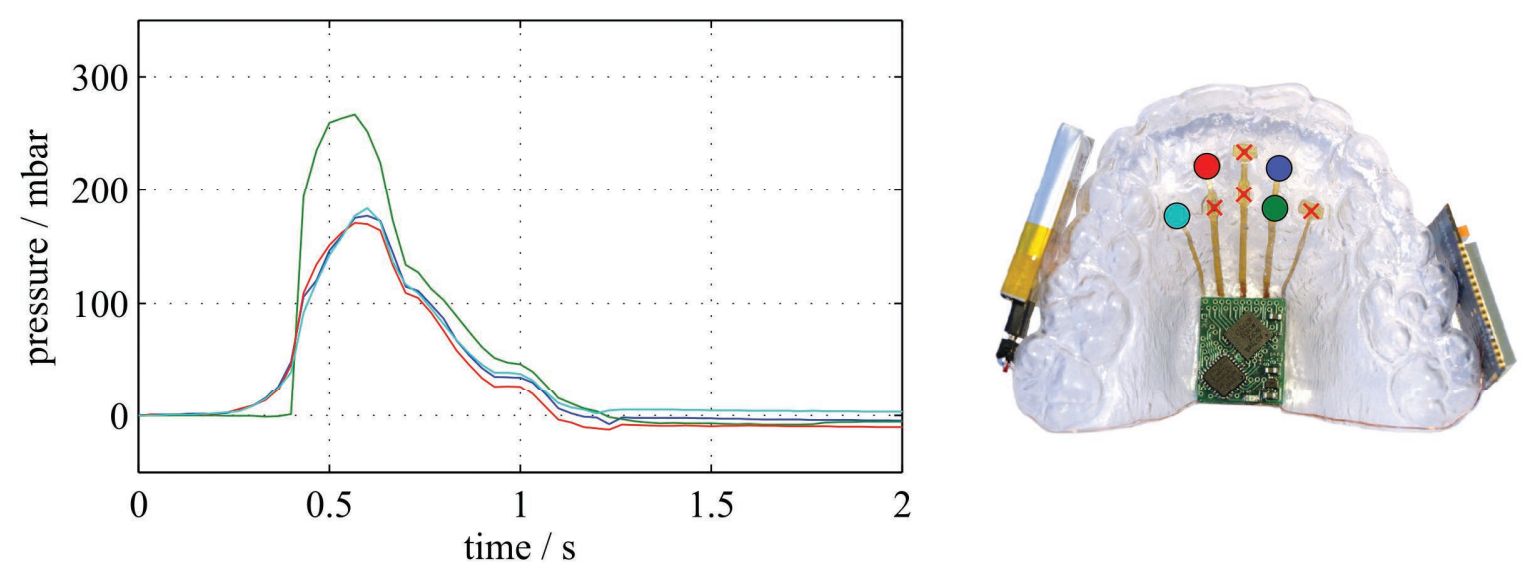

Fig. 4: Tongue pressure during swallowing of water. Colors represent the different sensor locations as shown on the right. Non functional sensors marked with red $X$.

\section{Conclusion and outlook}

A wireless measuring system for tongue pressure is designed and a first prototype presented. Through switching the excitation voltage of the resistive sensing elements the power consumption of the sensors could be reduced by $97 \%$. A very compact signal conditioning and data acquisition electronic with a noise free voltage resolution of $15.4 \mu \mathrm{V}$ and a power consumption of only $6.6 \mathrm{~mW}$ including the eight sensors is designed and integrated. Sampling rate is $30 \mathrm{~Hz}$ per sensor, but can be increased up to $150 \mathrm{~Hz}$ per sensor or even up to $4.8 \mathrm{kHz}$, if only one sensor is sampled. The overall power consumption with the ZigBit-module transmitting at $0 \mathrm{dBm}$ is $56 \mathrm{~mW}$, enabling the system to run over two hours from the build-in 60 mAh LiPo-battery.

First tests in the oral cavity show that the system is working as expected. Tongue pressure can be recorded and the wireless link is stable. Currently only four of the eight sensors are working properly. Here the mounting process has to be refined.

The originally designated wireless transceiver will allow a handsome reduction of size and power consumption. A specifically designed ASIC could help to reduce the power consumption of the sensors furthermore and lead to a smaller electronics. Due to the used standard processes for the PCB, the thickness of the electronics is still over $2 \mathrm{~mm}$. With a non-standard PCB or a flex PCB the thickness of a ready-to-market system can be reduced easily to less than $1.5 \mathrm{~mm}$. Further tests in the mouth have to prove long term stability and usability of the system.

\section{Literature}

[1] Müller, R.; Schuster, G.; Hohlfeld, O. et al.: Telemetrisches Messsystem zur Bestimmung des Druckes der Zunge gegen den Gaumen. Biomedizinische Technik. Vol. 48, 2003, S. 226-229.

[2] Stöhr, I.; Manderscheid, M.; Werthschützky, R.: Neuartiges polymerbasiertes Packaging von Silizium-Druckmesselementen zum Einsatz in der Kieferorthopädie. In: 9. Dresdner SensorSymposium, Dresden 2009

[3] Stöhr, I.; Werthschützky, R.: Polymer based packaging of silicon pressure sensors in the oral cavity. In: Sensor+Test Conference 2011, 7.-9. June 2011, Nürnberg. 